\title{
A PREDICTION TOOL FOR SPATIALLY DECAYING FREESTREAM TURBULENCE
}

\author{
Dwaipayan Sarkar, Eric Savory \\ Dept. of Mechanical \& Materials Engineering \\ The University of Western Ontario \\ London, Canada \\ dsarkar3@uwo.ca
}

\begin{abstract}
A set of new correlation equations characterizing the spatial decay of isotropic homogeneous turbulence has been formulated in the current study. The correlation equations are based on the results from the current numerical simulations combined with data from earlier relevant experimental studies [10], [15], [16]. The correlation equations can be effective for predicting the local values of turbulent properties (turbulence intensity (TI) and integral length scale $\left(\mathrm{L}_{\mathrm{u}}\right)$ ) from well specified initial conditions and, similarly, the magnitudes of inlet turbulence ( $\mathrm{TI}$ and $\mathrm{L}_{\mathrm{u}}$ ) can be estimated from the local magnitudes of length scale and turbulent kinetic energy (TKE) using the same prediction methodology. The set of prediction tools should be useful in the design of wind tunnel experiments to characterize bluff body aerodynamics in response to different incident turbulence conditions.
\end{abstract}

Keywords- isotropic, homogeneous, integral length scale, turbulence intensity

\section{INTRODUCTION}

The streamwise dissipation of isotropic, homogenous, free stream turbulence is one of the fundamental and widely explored topics in turbulence theory and its effects on various bluff body aerodynamics and thermal physics problems has been discussed over the past few decades [1-4]. In the absence of any external turbulent kinetic energy (referred to as TKE in the text hereafter) generation mechanism, the kinetic energy carried by the integral length scales of motion will decay, mostly due to inertial eddy interaction at high turbulent Reynolds number. Similarly, for low turbulent Reynolds number flows, molecular viscous forces dominate which causes the decay of TKE carried by the small scale dissipative eddies [1]. This decay of turbulent velocity fluctuations has an influential impact on the development of laminar and turbulent boundary layers over bluff bodies which in turn alters the heat and mass transfer rates from them [2-4]. Therefore, quantitative prediction of the free stream turbulent flow parameters incident on any bluff body, based on upstream inlet conditions, becomes an important factor for accurate determination of the dynamic properties of fluid and thermal responses in laminar, turbulent and transitional boundary layers.
There have been inconsistencies observed from the studies carried out for homogeneous and isotropic flows of freely decaying turbulence that have created disparities in the estimates of the spatial and temporal decay rates of TKE. Experimental grid-generated turbulence decays with a typical power law of the form, $\frac{\mathrm{k}}{\overline{\mathrm{U}}^{2}}=\mathrm{D} *\left(\frac{\mathrm{x}-\mathrm{x}_{0}}{\mathrm{M}}\right)^{-\mathrm{n}}$ [1], [5], where $\mathrm{k}$ is the TKE in the streamwise direction, $\bar{U}$ is the mean velocity, $D$ is the dimensionless dissipation constant, $\mathrm{x}$ is the streamwise distance, $\mathrm{x}_{0}$ is the virtual origin, $\mathrm{M}$ is the mesh width of the grid, and $\mathrm{n}$ gives the decay exponent. However, it was observed that different structures of geometrical grids generate turbulence with different decay rates and, hence, there is a need to achieve consistency, since the large-scale decay structures are known to affect the structure of turbulence at small scales [6], [7]. Numerous wind tunnel experiments have focused on generating homogeneous turbulence by different grids; passive [8], [9], active [10], [11], and multiscale (fractal) [12-15]. Numerical steady-state simulations [16] have also been carried out to reconcile the differences but no quantitative data have been presented at the inlet that would characterize the decay of turbulence downstream of a grid based on those specified initial conditions. A variation in the observed decay exponent $\mathrm{n}$ (from 1.0 to 1.4 ) implies that there might not be a universal state of decay mechanism.

It is generally agreed that the large scale turbulent properties, notably turbulence intensity (\%) (referred to as TI in the text, hereafter, given by $\left.\left(\mathrm{u}^{\prime} / \overline{\mathrm{U}}\right) \times 100\right)$ and integral length scale $\left(\mathrm{L}_{\mathrm{u}}\right)$ are the decisive factors governing the decay of quasi-static homogeneous isotropic turbulence, since both these parameters evolve according to power laws [1] and play an intrinsic role in dictating the dissipation rate of the TKE given by $\varepsilon \approx-\left(\mathrm{u}^{\prime 3} / \mathrm{L}_{\mathrm{u}}\right)$ , where $\mathrm{u}$ 'is the root-mean square of the characteristic turbulent velocity fluctuation and $\mathrm{L}_{\mathrm{u}}$ is the size of the average energy containing eddy. Therefore, the decay of free stream turbulence should be reflected in the values of $\mathrm{u}^{\prime}$ and $\mathrm{L}_{\mathrm{u}}$ at any point in the flow system and both parameters should be considered in the formulations that characterize the spatial decay of TKE. 
Hence, the aim of the current study is to employ Large Eddy Simulation (LES) models to develop simple yet powerful correlation equations that systematically quantify the spatial decay of turbulence, based on upstream inlet conditions, in a nearly isotropic and homogenous regime of turbulence. The final goal from the current study is to use those correlation functions as a prediction tool to estimate local and initial values of TKE and length scale which can then assist in numerous wind tunnel experiments of the flow over bluff bodies.

\section{METHODOLOGY}

\section{A. LES equations}

In LES the largest scales of turbulent motions are solved, whereas the smallest fluctuation scales are modelled. A spatial filtering is then applied to the continuity and the Navier-Stokes equations and the corresponding equations of the filtered velocity fields are obtained. The equations are then solved numerically using the concept of sub-grid viscosity $v_{\mathrm{t}}$ proposed by Smagorinsky [17], at each time-step, for each computational cell of the flow domain. More information about the numerical models associated with the LES formulation can be found in [18].

Among the sub-grid scale models that are available for LES, the dynamic sub-grid scale model by Smagorinsky [17], and Germano et al. [19] was used in the present work for simulating spatially decaying homogeneous isotropic turbulence.

\section{B. Solution parameters}

Different solution parameters used for handling the flow variables in LES are summarized in Table I. In order to maintain the convergence and the accuracy of the solution, the Courant Fredrichs-Lewy (CFL) number [20] is maintained at less than 1.0 by setting the time-step accordingly, based on the gridspacing. The LES simulations were carried out for 20,000 timesteps and statistically averaged results are presented. The simulations were performed using SHARCNET [21] a highcomputing cluster facility available at Western University.

TABLE I. Solution Parameters used in LES

\begin{tabular}{|c|c|}
\hline Time discretization & Second order implicit \\
\hline Momentum discretization & Bounded central difference \\
\hline Pressure discretization & Second order \\
\hline Pressure-velocity coupling & Segregated \\
\hline Under relaxation factors & $\begin{array}{c}0.5 \text { for the velocity and } 0.2 \\
\text { for the pressure }\end{array}$ \\
\hline
\end{tabular}

\section{CFD MODELLING DETAILS}

\section{A. Computational Domain and Boundary Conditions}

A three dimensional (3D) computational domain was created with sufficient length in the streamwise direction to allow for spatial decay of turbulence to develop fully. The geometry, along with the mesh, was generated using ICEM v.16.0 [22].
The domain was discretized using a perfect structured hexahedral mesh in all the three directions for accurate interpolation of the mean flow quantities and to be consistent with the cut-off scales for the spatial filtering. The physical dimensions of the grid were $L_{x}=4 \mathrm{~m}, L_{y}=1 \mathrm{~m}, L_{z}=1 \mathrm{~m}$.

Generating a proper inflow boundary condition for LES simulations is important [23-25] as the inflow should maintain proper spectra and coherency to replicate the spatial turbulent flow characteristics throughout the domain. Hence, to develop an isotropic homogeneous inflow boundary condition, the Consistent Random Flow Generation (CDRFG) technique was used. More information about this technique can be found in [26]. Pure slip wall conditions with zero shearing were imposed at the walls to negate the effect of the physical boundaries on the decay of TKE. A pressure outlet was specified as a suitable boundary condition for the outlet boundary. The parameters for generating the inlet velocity conditions are summarized in Table II.

TABLE II. Parameters used for generating the inlet velocity field

\begin{tabular}{|l|c|}
\hline Parameters & Values \\
\hline Mean velocity & $4 \mathrm{~m} / \mathrm{s}$ \\
\hline Integral length scale & $0.1 \mathrm{~m}$ \\
\hline Turbulence intensity & $10 \%$ \\
\hline Cut-off frequency & $100 \mathrm{~Hz}$ \\
\hline No. of samples generated & 20000 \\
\hline
\end{tabular}

\section{B. Grid-Independency}

Three different grid resolutions M1, M2 and M3 were created and used for the present grid-independence tests. The properties of these three grids are summarized in table III. The grid-independence study was carried out for a mean inlet velocity $(\bar{U})$ of $4 \mathrm{~m} / \mathrm{s}$, initial TI of $10 \%$ and integral length scale $\left(\mathrm{L}_{\mathrm{u}}\right)$ of $0.1 \mathrm{~m}$.

TABLE III. Properties of the employed grids in the current study

\begin{tabular}{|c|c|c|c|}
\hline Grid & Type & Element size $(\boldsymbol{\Delta x})$ & No. of cells \\
\hline M1 & Coarse & $40 \mathrm{~mm}$ & 250,000 \\
\hline M2 & Medium & $20 \mathrm{~mm}$ & 500,000 \\
\hline M3 & Fine & $10 \mathrm{~mm}$ & $1,000,000$ \\
\hline
\end{tabular}

For LES, the variability in non-dimensional TKE going from M1 to M2 grid in proportion to its average value over the constant region where the TKE becomes fairly uniform (i.e. $\mathrm{x}=2 \mathrm{~m}$ to $\mathrm{x}=4 \mathrm{~m}$ ) is $13.1 \%$, whereas the variability of the same variable going from $\mathrm{M} 2$ to $\mathrm{M} 3$ is $4.8 \%$. On a similar note, the variation of the magnitude of $\mathrm{L}_{\mathrm{u}}$ in proportion to its average value over the constant region from M1 to M2 is 5.9\% and from $\mathrm{M} 2$ to M3 it is $2.3 \%$.

Hence, the grid (M3) with 1,000,000 cells was chosen for the LES computations, corresponding to the least relative discretization error with an acceptable value of convergence [27]. The employed grid also resolves the most energy 
containing eddies (ensuring that $80 \%$ of the TKE is resolved in the present case).

\section{Validation of the current CFD model}

The time-averaged, non-dimensional TKE profiles along the centreline of the domain in the streamwise direction using the Dynamic-Smagorinsky LES model is compared with the previous existing experimental and numerical data of [10], which are more recent than the classical Comte-Bellot and Corrsin experiments [28], [29] in high turbulent Reynolds number flows (fig. 1). It is clear from figure (1) that the quantitative agreement between the LES and different sets of data presented from that study [10] is remarkably good with a proper choice of virtual origin $\mathrm{x}_{0}$ value (which refers to the distance downstream of an experimental grid origin, where the wake vortices from the grid has decayed sufficiently and the turbulence has started to become well-developed and nearly isotropic and homogeneous in nature). It should be noted that the choice of the virtual origin is sensitive to the decay rate of TKE and, in this comparison, the virtual origin of the experiments was typically taken at 20 mesh widths i.e. $\mathrm{x}_{0}=20 \mathrm{M}$, for comparison [1].

The agreement between these two studies show that in the absence of mean shear and boundary layer wall effects, welldeveloped turbulent flow decays at a similar rate and there exists a universal decay mechanism of turbulence that would fit a single non-dimensional curve.

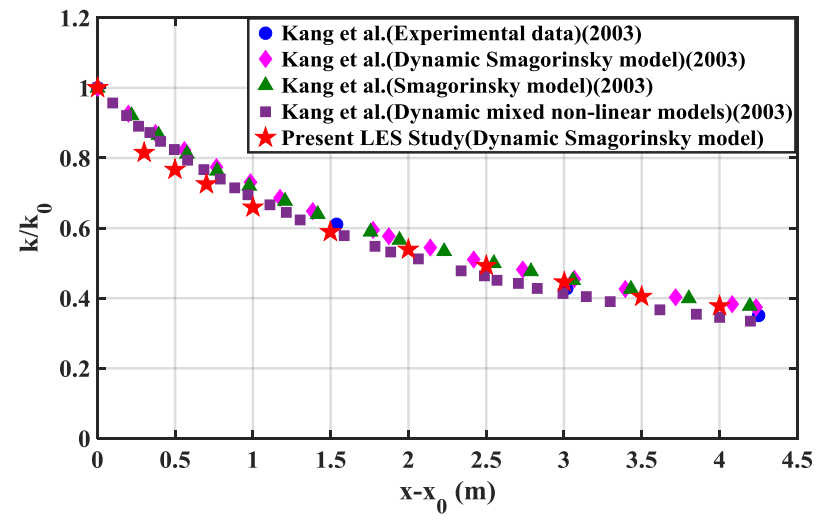

Figure 1. Comparison of decay of TKE in the streamwise direction between the studies of Kang et al. (2003) and present LES results.

Further comparison between the two studies reveals that the magnitude of $\frac{\mathrm{dk}^{\prime}}{\mathrm{dx}}$ at the virtual origin $\left(\mathrm{x}_{0}\right)$, computed from the values of $\mathrm{D}, \mathrm{u}^{\prime}, \overline{\mathrm{U}}$ and $\mathrm{L}_{\mathrm{u}}$ gives an approximate value of $0.014 \mathrm{~m}^{-}$ ${ }^{1}$ for the experiments [10] and $0.010 \mathrm{~m}^{-1}$ for the present LES study which are close to each other. Since the initial spatial TKE gradient rate controls the downstream decay of the TKE, a similar tendency of its decay in the nearly isotropic and homogeneous region of turbulence is observed.

\section{RESUlTS \& DISCUSSIONS}

\section{A. Predictive Methods for Spatial Decay of TKE}

A new predictive algorithm based on the current methodology has been formulated in the present study which provides an estimate of the local and initial turbulence levels in the uniform flow approaching the leading edge of a bluff body. The predictive algorithm is based on the simple inviscid estimation of the turbulence dissipation rate $\varepsilon$ at a given length scale, represented by $\varepsilon=\mathrm{dk} / \mathrm{dt}$. Starting from the inviscid estimation of $\varepsilon$ given by $\varepsilon=-\mathrm{D}\left(\mathrm{u}^{\prime 3} / \mathrm{L}_{\mathrm{u}}\right.$ ) (where $\mathrm{D}$ is the dimensionless dissipation constant, taken here as being unity, for convenience) and invoking Taylor's hypothesis of frozen turbulence, both these forms of $\varepsilon$ can be related and a new power law equation derived (which includes both TKE and $\mathrm{L}_{\mathrm{u}}$ ) that gives the spatial variation of TKE in the streamwise direction presented in the form

$$
\frac{\mathrm{k}}{\mathrm{k}_{0}}=\frac{\mathrm{k}^{\prime}}{\mathrm{k}_{0}^{\prime}}=\left(\frac{\mathrm{A}\left(\mathrm{k}_{0}{ }^{\prime}\right)^{0.5} \mathrm{x}}{\mathrm{L}_{\mathrm{u}}}+1\right)^{-\mathrm{n}}
$$

where $\mathrm{k}$ and $\mathrm{k}_{0}$ are the local and initial TKE (at $\mathrm{x}=0$ ), $\mathrm{k}^{\prime}$ and $\mathrm{k}_{0}{ }^{\prime}$ are the dimensionless local and initial TKE (at $\mathrm{x}=0$; $\mathrm{k}$ is normalized using the mean velocity $\overline{\mathrm{U}}, \mathrm{A}$ is a dimensionless constant having a magnitude of 0.27 (where $\mathrm{A}=0.27 \times \mathrm{D} ; \mathrm{D}=1$ ), $\mathrm{n}$ is the decay exponent of magnitude $2, \mathrm{x}(\mathrm{m})$ is the streamwise distance starting from the origin of the physical grid and $\mathrm{L}_{u}$ is the integral length scale (m) which is taken as a constant in this derivation for simplicity. This correlation equation (1) assumes turbulence is strictly isotropic and Taylor's hypothesis has been invoked while deriving spatial functions of the velocity field. However, in reality, the integral length scale $\left(\mathrm{L}_{\mathrm{u}}\right)$ grows in the streamwise direction as turbulence decays and cannot be treated as a constant which then alters the magnitude of the power law exponent, $\mathrm{n}$, obtained from the correlation function presented in equation (1). Hence, to incorporate the effects of the spatial evolution of the length scale $\left(\mathrm{L}_{\mathrm{u}}\right)$, the correlation equation (1) is rewritten in a general form as

$$
\frac{\mathrm{k}}{\mathrm{k}_{0}}=\frac{\mathrm{k}^{\prime}}{\mathrm{k}_{0}^{\prime}}=\left(\frac{\mathrm{A}_{1}\left(\mathrm{k}_{0}^{\prime}\right)^{0.5}\left(\mathrm{x}-\mathrm{x}_{0}\right)}{\mathrm{L}_{\mathrm{u}}}+1\right)^{-\mathrm{n}_{1}}
$$

where $\mathrm{x}_{0}$ is the virtual origin, and $\mathrm{A}_{1}, \mathrm{n}_{1}$ are the decay coefficient and decay exponent, respectively, for the above correlation (eq. 2).

Replacing $\mathrm{L}_{\mathrm{u}}$ with $\mathrm{L}_{\mathrm{u} 0}$ another form of decay power law can be achieved with the following form

$$
\frac{\mathrm{k}}{\mathrm{k}_{0}}=\frac{\mathrm{k}^{\prime}}{\mathrm{k}_{0}{ }^{\prime}}=\left(\frac{\mathrm{A}_{2}\left(\mathrm{k}_{0}{ }^{\prime}\right)^{0.5}\left(\mathrm{x}-\mathrm{x}_{0}\right)}{\mathrm{L}_{\mathrm{u} 0}}+1\right)^{-\mathrm{n}_{2}}
$$

where $L_{u 0}$ is the initial value of the integral length scale (at $x=$ $0), A_{2}$ and $n_{2}$ are the new decay coefficient and decay exponent, respectively, for the above correlation equation (3).

The correlations presented in equations $(2,3)$ clearly depict a power law form analogous to the grid-generated TKE decay law given in [1].

The correlation equation obtained in equation (2) is tailored specifically to include the effects of the TKE and the integral length scale into a single correlation equation, since the 
evolution of length scales influence the energy decay of the turbulent velocity fluctuations [9], [30].

The range of decay exponent (n) values obtained for different types of grids and numerical simulations is large because it depends on the initial conditions of the flow, the physical characteristics of the experimental grids (shape, size, width and porosity) and the choice of the virtual origin $\left(\mathrm{x}_{0}\right)$ [7], [31]. A faster or slower rate of decay of TKE is also possible depending upon the solidity of the grid which depends on the porosity $(\beta)$ which, in turn, is a function of the rod or wire dimeter used and the mesh width (M), as reported in [6].

Hence, the present work does not aim to address the question of determining definitive and precise values of the decay exponent (n) exhibited in eq. (2), but, rather, to come up with a global decay exponent (n) and dimensionless constant (A), that best fits the correlation function when using all the relevant previous experimental studies and the present numerical simulations, within an acceptable degree of accuracy, in the region of well-developed, homogeneous and isotropic turbulence.

The correlation (eq.2 and eq. 3) can be used to obtain an estimate of the local statistical turbulence parameters $\left(\mathrm{k}\right.$ and $\left.\mathrm{L}_{\mathrm{u}}\right)$ at any location downstream of a grid based on the specified initial turbulence conditions $\left(\mathrm{k}_{0}\right.$ and $\left.\mathrm{L}_{\mathrm{u} 0}\right)$ at the inlet. Similarly, the same set of prediction equations (2 and 3) also helps to recover the initial free-stream turbulence levels relevant to any bluff-body aerodynamics problem knowing the local magnitudes of turbulence scales, thus making this prediction method a complete closure solution to the choice of experimental turbulence generating grid required to achieve a particular level of turbulence.

\section{B. Prediction equation based on the local value of the integral length scale $\left(L_{u}\right)$}

Figure (2) shows the direct comparisons between the earlier experimental studies of Kang et al. (2003) [10], Krogstad and Davidson (2011) [15], Torrano et al. (2015) [16] and the present LES with a best fit curve for the correlation function presented in equation (2). The streamwise evolution of non-dimensional TKE profiles $\left(\mathrm{k} / \mathrm{k}_{0}\right)$ are plotted against a non-dimensional parameter $\left(\mathrm{x}-\mathrm{x}_{0}\right) *\left(\mathrm{k}_{0}\right)^{0.5} / \mathrm{L}_{\mathrm{u}}$ where, $\left(\mathrm{x}-\mathrm{x}_{0}\right)$ is

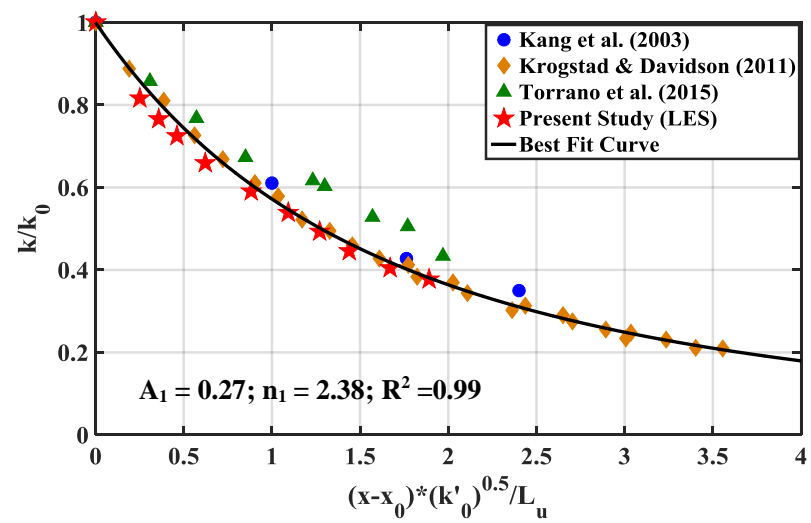

Figure 2. Spatial decay of TKE profiles of earlier experimental studies and present LES , plotted with a solid line that shows the best-fit power law the effective origin, $\left(\mathrm{k}_{0}{ }^{\prime}\right)$ is the non-dimensional initial TKE value and $\left(\mathrm{L}_{\mathrm{u}}\right)$ is the local value of the integral length scale. The magnitude of the initial normalized TKE and integral length scale values are digitized and extracted from the previous studies to achieve a direct comparison between the variables used in the plots by using the above scaling, even though the initial conditions of the different experiments and the LES are different. The fitted coefficient values of $\mathrm{A}$ and $\mathrm{n}$ estimated from the best-fit curve are $\left(A_{1}=0.27\right.$ and $\left.n_{1}=2.38\right)$. The goodness of the fit is excellent at $\mathrm{R}^{2}=0.99$. Figure (4) also reveals that the numerical results are fairly consistent (within $3 \%$ ) with the experimental data and both the studies reproduce a similar tendency of the predicted turbulence energy decay.

\section{Prediction equation based on the initial value of the integral length scale $\left(L_{u 0}\right)$}

Figure (3) shows the comparison of the spatial decay of the TKE profiles for different experiments stated earlier and the LES study, with the $\mathrm{x}$ axis variable $\left(\mathrm{x}-\mathrm{x}_{0}\right) *\left(\mathrm{k}_{0}\right)^{0.5} / \mathrm{L}_{\mathrm{u} 0}$ now normalized with the initial constant value of $\mathrm{L}_{\mathrm{u}}$, i.e. $\left(\mathrm{L}_{\mathrm{u} 0}\right)$, instead of local values of $\mathrm{L}_{\mathrm{u}}$. The scaling parameter has been changed to see its effect on the quantitative agreement between the LES and the experimental studies. Here $\mathrm{R}^{2}=0.97$, which is not as good as the previous scaling, whilst the values of $\mathrm{A}_{2}$ and $\mathrm{n}_{2}$ are 0.44 and 1.16 , respectively. It turns out that the $\mathrm{A}_{2}$ and $\mathrm{n}_{2}$ values obtained from the regression fit (eq. 3) have different

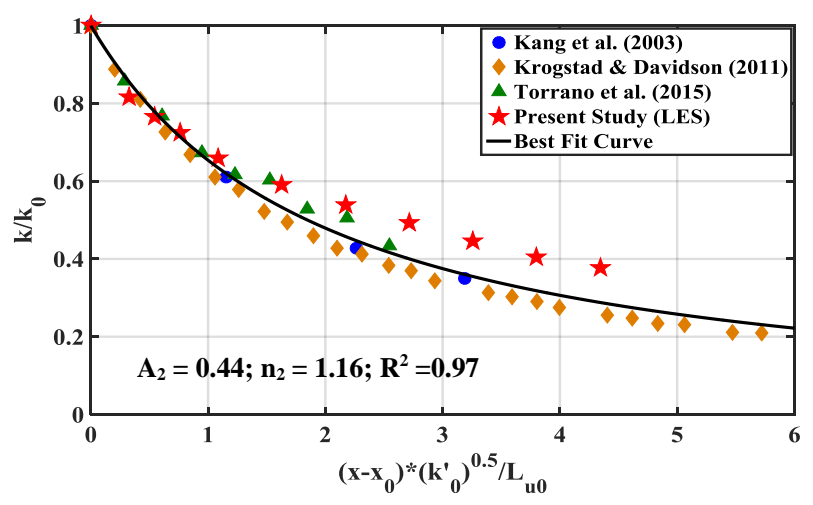

Figure 3. Spatial decay of TKE profiles for different experiments and the present LES study scaled with initial integral length scale $\left(\mathrm{Lu}_{0}\right)$ plotted with a solid line that shows the best-fit power law

magnitudes compared to the $\mathrm{A}$ and $\mathrm{n}$ obtained while deriving equation (1) even though both correlations assume a constant $\mathrm{L}_{\mathrm{u}}$. The values of the decay coefficient $\mathrm{A}$ and the decay exponent $\mathrm{n}$ obtained from equation (1) are 0.27 and 2 respectively.

There are reasons for this observed variation, between the magnitudes of decay coefficient $\mathrm{A}$ and decay exponent $\mathrm{n}$, obtained from the simple inviscid estimation of $\varepsilon$ (eq. 1) and the best-fit curve over all the experimental data and LES results (eq. $3)$.

Firstly, strictly isotropic turbulence has been assumed while deriving the correlation (eq. 1), which in reality is difficult to 
achieve in turbulence generated in computer simulations [32]. However, this is not surprising as such inhomogeneity of the flow has already been observed in the earlier experiments of [33], [34]. Secondly, while deriving the correlations presented in equations (1), (2) and (3), it was assumed that D is a nondimensional constant of magnitude unity, having no temporal or spatial dependency in the streamwise direction, but $\mathrm{D}$ is seen to have spatial dependency in the studies reported in [9]. Therefore, the estimate of $A_{2}$ and $n_{2}$ obtained from the best fit curve shown in figure (3) inherently incorporates the variability of $\mathrm{D}$ if any, present in the current simulations.

\section{Prediction equation based on the local magnitude of TKE \\ $(k)$ and local magnitude of integral length scale $\left(L_{u}\right)$}

Figure (4) shows another form of the spatial decay of TKE profiles from both the experiments and LES data, but with the $\mathrm{x}$ ordinate scaled with the local non-dimensional TKE, $\mathrm{k}$, and the local magnitude of the integral length scale $\left(\mathrm{L}_{\mathrm{u}}\right)$. Figure (6) reveals a linear relationship between $\left(\mathrm{k} / \mathrm{k}_{0}\right)$ and $\left(\mathrm{x}-\mathrm{x}_{0}\right) *\left(\mathrm{k}^{\prime}\right)^{0.5} / \mathrm{L}_{\mathrm{u}}$ which implies

$$
\frac{\frac{\mathrm{d}}{\mathrm{dx}}\left(\mathrm{k} / \mathrm{k}_{0}\right)}{\frac{\mathrm{d}}{\mathrm{dx}}\left(\mathrm{x}-\mathrm{x}_{0}\right)^{\prime}\left(\mathrm{k}^{\prime}\right)^{0.5} / \mathrm{L}_{\mathrm{u}}} \approx \mathrm{C}
$$

This holds true and is manifest through the power law dependency of $\mathrm{k}$ and $\mathrm{L}_{\mathrm{u}}$ along the streamwise direction which is given by the following equation

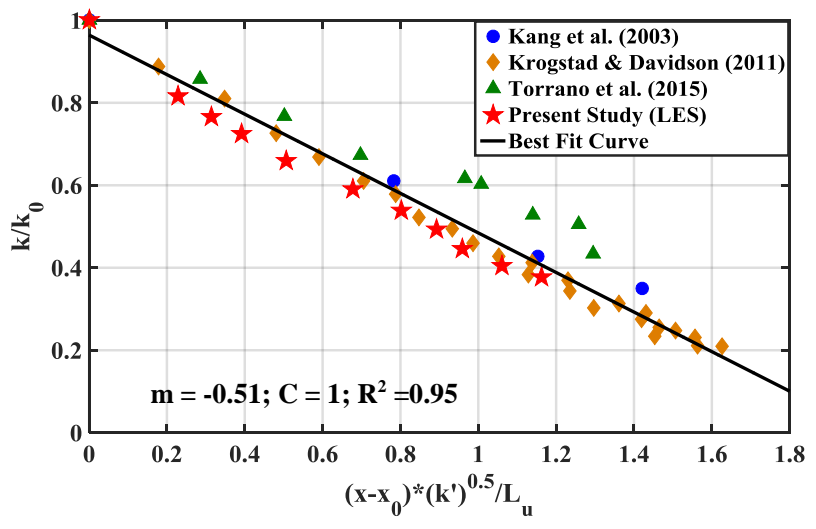

Figure 4. Spatial decay of TKE profiles for different experiments and the present LES data with the $\mathrm{x}$ ordinate scaled with local $\mathrm{k}$ ' and $\mathrm{L}_{\mathrm{u}}$

$\frac{\mathrm{k}}{\mathrm{k}_{0}}=\frac{\mathrm{k}^{\prime}}{\mathrm{k}_{0}^{\prime}}=\left(\mathrm{a}_{1} \mathrm{x}+1\right)^{-\mathrm{p}}$

$\frac{\mathrm{L}_{\mathrm{u}}}{\mathrm{L}_{\mathrm{u} 0}}=\left(\mathrm{a}_{2} \mathrm{x}+1\right)^{\mathrm{q}}$

In order to arrive at linearity; $\left(\mathrm{a}_{1} \approx \mathrm{a}_{2}\right)$ and a slight deviation from the numbers can affect the linearity of the experimental data sets and the LES results seen in figure (4), which is simply a consequence of the curve fitting performed for the best fit for equations (11), (12). Similarly, $\mathrm{p}$ and $\mathrm{q}$ are closely related to each other in the form of $q / 2-p=1$ for the LES results, which is verified by the exponents derived in [9]. The left-hand side of equation (4) can be then solved to arrive at a constant value. The best linear fit line is drawn in the figure which gives a $R^{2}=0.96$ with the slope $m$ being -0.51 and the intercept $C$ being 1 . Hence the final equation of the spatial decay of TKE stands in the form of

$\left(\frac{\mathrm{k}}{\mathrm{k}_{0}}\right)=\left(\frac{\mathrm{k}^{\prime}}{\mathrm{k}_{0}^{\prime}}\right)=\mathrm{m}\left(\frac{\left(\mathrm{x}-\mathrm{x}_{0}\right)^{*}\left(\mathrm{k}^{\prime}\right)^{0.5}}{\mathrm{~L}_{\mathrm{u}}}\right)+\mathrm{C}$

It is worthwhile mentioning that the correlation functions presented in equation (3) along with equation (7) also helps one to predict the initial TKE $\left(\mathrm{k}_{0}\right)$ and length scale values $\left(\mathrm{L}_{\mathrm{u}}\right)$, provided one knows the local values of TKE (k) and length scale $\left(\mathrm{L}_{\mathrm{u}}\right)$, thus giving another method of estimating the statistical turbulence parameters in the domain.

There are, altogether, 3 sets of data, which have been used here for the predictive methodology, in accordance with the previous experimental studies, to estimate the magnitude of the TKE and $\mathrm{L}_{\mathrm{u}}$ values. The values of the fitted coefficients for all sets of data are summarized in table IV and V.

TABLE IV Constants obtained from best regression curve fitting procedure using the method of non-linear least squares

\begin{tabular}{|c|c|c|c|c|}
\hline $\begin{array}{c}\text { Normalization } \\
\text { parameter of the } \\
\mathbf{x} \text { ordinate }\end{array}$ & $\mathbf{R}^{2}$ & $\begin{array}{c}\text { Decay } \\
\text { coefficient } \\
(\mathbf{A})\end{array}$ & $\begin{array}{c}\text { Decay } \\
\text { exponent } \\
(\mathbf{n})\end{array}$ & $\begin{array}{c}\text { Equation } \\
\text { number }\end{array}$ \\
\hline $\mathrm{k}_{0}{ }^{\prime}$ and $\mathrm{L}_{\mathrm{u}}$ & 0.99 & 0.27 & 2.38 & 2 \\
\hline $\mathrm{k}_{0}{ }^{\prime}$ and $\mathrm{L}_{\mathrm{u} 0}$ & 0.97 & 0.44 & 1.16 & 3 \\
\hline
\end{tabular}

TABLE V Constants obtained from best regression curve fitting procedure using the method of non-linear least squares

\begin{tabular}{|c|c|c|c|c|}
\hline $\begin{array}{c}\text { Normalization } \\
\text { parameter of the } \\
\mathbf{x} \text { ordinate }\end{array}$ & $\mathbf{R}^{\mathbf{2}}$ & $\mathbf{m}$ (slope) & $\begin{array}{c}\text { C (intercept } \\
\text { on the } \mathbf{y} \\
\text { ordinate) }\end{array}$ & $\begin{array}{c}\text { Equation } \\
\text { number }\end{array}$ \\
\hline $\mathrm{k}^{\prime}$ and $\mathrm{L}_{\mathrm{u}}$ & 0.95 & -0.51 & 1 & 4 \\
\hline
\end{tabular}

It is worth mentioning that these correlations $(2,3)$, while written in logarithmic form along with the correlation given by equation (7), each represent a simple linear form of equations involving one known and one unknown variable which can then be used to estimate either the local turbulence parameters at any downstream location or to predict the initial turbulent parameters for any turbulent flow. Thus, the equations (2), (3) and (7) complete the prediction tool for designing turbulence generating grids. More information about the applicability of these prediction equations can be found in [35].

\section{CONCLUSIONS}

The present work attempts to simulate freely decaying isotropic homogenous turbulence using Large Eddy Simulation (LES). The current results show good quantitative agreement with the previous experimental results from [10], [15], [16]. Three different correlations were formulated which can be useful in estimating the local and initial values of turbulent parameters (i.e. TKE and $\mathrm{L}_{\mathrm{u}}$ ) which can then aid the design of wind tunnel experiments. 


\section{ACKNOWLEDGMENT}

This work was made possible by the facilities of the Shared Hierarchical Academic Research Computing Network (SHARCNET: www.sharcnet.ca) and Compute/Calcul Canada.

\section{REFERENCES}

[1] Pope, S. B. Turbulent Flows. Cambridge University Press (2000).

[2] J.C. Simonich and P. Bradshaw, "Effect of free stream Turbulence on Heat transfer through a Turbulent Boundary Layer" Journal of Heat Transfer, ASME, Vol.100, pp. 671-677 (1978).

[3] M.F. Blair, "Influence of Free Stream Turbulence on Turbulent Boundary Layer Heat transfer and Mean Profile Development, Part-I-Experimental data, Journal of Heat Transfer, Vol. 105, pp. 33-40 (1983).

[4] M.F. Blair, "Influence of Free Stream Turbulence on Turbulent Boundary Layer Heat transfer and Mean Profile Development, Part-II-Experimental data, Journal of Heat Transfer, Vol. 105, pp. 41-47 (1983).

[5] G.K. Batchelor and A.A. Townsend, "Decay of isotropic Turbulence in the initial period", Proceedings of the Royal Society of London, Series A, Mathematical and Physical Sciences, Vol. 193, Issue 1035, pp. 539-558 (1948).

[6] J Tan-Atichat, H M Nagib and R I Loehrke, "Interaction of free-stream turbulence with screens and grids: a balance between turbulence scales" J. Fluid Mech. Vol. 114, pp.501-28 (1982).

[7] P Lavoie, P Burattini, L Djenidi and R Antonia, "An Effect of initial conditions on decaying grid turbulence at low $\mathrm{R}_{\lambda}$ " Exp. Fluids Vol. 39, pp.865-874 (2005).

[8] T Ishida, P.A. Davidson, and Y. Kaneda, "On the decay of isotropic turbulence". Journal of Fluid Mechanics, 564, Sept., pp. 455-475 (2006).

[9] P.A. Krogstad, and P.A. Davidson, "Is grid turbulence Saffman turbulence?". Journal of Fluid Mechanics, Vol. 642, Dec., p. 373-394 (2009).

[10] H.S. Kang, S. Chester, and C. Meneveau, "Decaying turbulence in an active-grid-generated flow and comparisons with large-eddy simulation". Journal of Fluid Mechanics, Vol. 480, Apr., pp. 129-160 (2003).

[11] N. Mordant, "Experimental high Reynolds number turbulence with an active grid". American Journal of Physics, Vol. 76(12), p. 1092-1098 (2008).

[12] B. Mazzi, and J.C. Vassilicos, "Fractal-generated turbulence". Journal of Fluid Mechanics, Vol. 502(1), pp. 65-87 (2004).

[13] R.E. Seoud, and J.C. Vassilicos, "Dissipation and decay of fractalgenerated turbulence". Physics of Fluids, Vol. 19(10), pp. 105-108 (2007).

[14] D. Hurst, and J.C. Vassilicos, "Scalings and decay of fractal-generated turbulence". Physics of Fluids, 19(3), Mar., pp. 1-31 (2007).

[15] P.A. Krogstad, P.-A., and P.A. Davidson, "Freely decaying, homogeneous turbulence generated by multi-scale grids". Journal of Fluid Mechanics, 680, pp. 417-434 (2011).

[16] I. Torrano, M. Tutar, M. M-Agirre, A. Rouquier, N. Mordant and M. Bourgoin, "Comparison of Experimental and RANS-Based Numerical Studies of the Decay of Grid-Generated Turbulence", Journal of Fluids Engineering, Vol. 137 (6), pp. 1-12, (2015).
[17] J. Smagorinsky General circulation experiments with the primitive equations: I. The basic experiment. Mon Weather Rev; 91:99-164 (1963).

[18] Ansys Inc.,. ANSYS FLUENT Theory Guide, pp. 1-814 (2005).

[19] M Germano, U Piomelli, P Moin, WH Cabot, "A dynamic subgrid-scale eddy viscosity mode"l. Phys Fluids; Vol. 3(7), pp.1760-1765 (1991).

[20] Courant, R., Friedrichs, K. and Lewy, H.,. On the partial difference equations of mathematical physics. IBM journal of Research and Development, 11(2), pp. 215-234. (1967).

[21] SHARCNET: a consortium of colleges, universities and research institutes operating a network of high-performance computer clusters across southwestern, central and northern Ontario [Online]. Available: www.sharcnet.ca (2015).

[22] ICEM v.16.0. Ansys Product. < http://www.ansys.com> (2016).

[23] J-L Aider, A. Danet "Large-eddy simulation study of upstream boundary conditions influence upon a backward-facing step flow". C.R. Mecanique; Vol. 53 (334), pp. 447-453, (2006).

[24] P. Sagaut, E. Garnier, E. Tromeur, L. Larcheveque, E. Labourasse, "Turbulent in-flow conditions for LES of subsonic and supersonic wallbounded flows", Am. Inst. Aeronautic. Astronaut. J., Vol. 42, pp. 469-478 (2003).

[25] G.R. Tabor, M.H. Baba-Ahmadi, "Inlet conditions for large eddy simulation: A review", "Computers \& Fluids", Vol. 39, pp. 553-567 (2010).

[26] H. Abasosha, A. Elshaer, G. Bitsuamlak, A. El. Dalmatty, "Consistent inflow turbulence generator for LES evaluation of wind-induced responses for tall buildings", Journal of Wind Engineering and Industrial Aerodynamics, Vol. 142, pp. 198-216 (2015).

[27] Georgiadis, N.J., Rizzetta, D.P. and Fureby, C., "Large-eddy simulation: current capabilities, recommended practices, and future research". AIAA journal, 48(8), pp.1772-1784 (2010).

[28] Comte-Bellot, G. and Corrsin, S., "The use of a contraction to improve the isotropy of grid-generated turbulence". Journal of fluid mechanics, 25(4), pp.657-682 (1966).

[29] Comte-Bellot, G. and Corrsin, S., Simple Eulerian time correlation of fulland narrow-band velocity signals in grid-generated, 'isotropic'turbulence. Journal of Fluid Mechanics, 48(2), pp.273-337 (1971).

[30] Dryden, H.L., 1943. "A review of the statistical theory of turbulence". Quarterly of Applied Mathematics, 1(1), pp.7-42 (1943).

[31] M S Mohamed M S and J C LaRue, "The decay power law in gridgenerated turbulence" J. Fluid Mech. 219, pp. 195-214 (1990).

[32] Ishida, T., Davidson, P.A. and Kaneda, Y., "On the decay of isotropic turbulence". Journal of Fluid Mechanics, 564, pp.455-475 (2006).

[33] Bennett, J.C. and Corrsin, S., "Small Reynolds number nearly isotropic turbulence in a straight duct and a contraction". The Physics of Fluids, 21(12), pp.2129-2140 (1978).

[34] Lavoie, P., Djenidi, L. and Antonia, R.A., "Effects of initial conditions in decaying turbulence generated by passive grids". Journal of Fluid Mechanics, 585, pp.395-420 (2007).

[35] Sarkar, D, "A numerical tool for predicting the spatial decay of freestream turbulence.”. MESc thesis, Univ W Ontario, Electronic Thesis and Dissertation Repository 5331 (2018). 